\title{
THE EFFECT OF FIRMS REPORTING TO THE CARBON DISCLOSURE PROJECT ON THEIR CO, EMISSIONS: APPLICATION ON INDUSTRIAL SECTOR
}

\author{
A. TURKOVÁ \& L. DONZE \\ Applied Statistics and Modelling, Department of Informatics, University of Fribourg, Switzerland.
}

\begin{abstract}
In this article we investigate the impact of the Carbon Disclosure Project, as one of the binding reporting standards on a firms' emissions. In particular, we focus on firms from the industrial sector and analyse whether reporting to Carbon Disclosure Project has a positive effect and leads the firm to reduce their $\mathrm{CO}_{2}$ emissions. In order to evaluate this effect we use a relatively new method called 'synthetic control approach', which allows us not only to measure the impact of the firms' policy, but also to evaluate the significance of our estimates. Based on a unique database we constructed, we found that with the exception of three firms, there was no significant effect on the firms' emissions.

Keywords: carbon disclosure project, $\mathrm{CO}_{2}$ emissions, program evaluation, synthetic control methods.
\end{abstract}

\section{INTRODUCTION}

Nowadays, we are more aware of climate change and so increasingly sensitive to the 'green' economics in order to assure the future. Consequently, companies are pushed to rapidly and significantly cut down their $\mathrm{CO}_{2}$ emissions and review their policies in this direction. The objective of our study is to assess the pertinence of green policy introduction at the business level. For our analysis, we are using unique data sets of the firm's $\mathrm{CO}_{2}$ emissions. We built our data by adding several firms' characteristics to an initial database provided by South Pole Company. We intend to evaluate whether signing up to the Carbon Disclosure Project (CDP), as one of the binding reporting standards, has a positive effect on the firms emissions. It is a typical causal effect evaluation problem that we solve using a relatively new method called 'synthetic control approach' introduced by Abadie and Gardeazabal [1]. This approach is an econometric method used for program or treatment evaluation. Almer and Winkler [2] used this method in environmental problematic, but to our knowledge it has not been applied to evaluate a firms' policy such as environmental programme at a company level. We chose this method because it allows researchers to analyse phenomena that occurs in a limited population or that apply to only a small number of firms, which is perfectly suited to our problematic. Furthermore, this method allows to perform inference analysis and support quantitatively the results.

In this paper we concentrate on the industrial sector, and in particular on companies from the European Union, United Kingdom and United States that started to report in years 2009 and 2010. The companies $\mathrm{CO}_{2}$ emissions include Scope 1 and 2 greenhouse gas emissions. And in order to normalise the $\mathrm{CO}_{2}$ emissions we consider the $\mathrm{CO}_{2}$ emissions per employee as a main outcome variable to be studied. 


\subsection{Carbon disclosure project}

CDP is an international non-profit organisation founded in 2003 and based in the United Kingdom. CDP's objective is to help institutions to work on the strategies that improve the management of environmental risk, as reduction of $\mathrm{CO}_{2}$ emissions and use of energy. Different to the Kyoto protocol, CDP focuses on individual companies rather than a nation. Today, CDP works with 827 institutional investors, government and policymakers holding US\$100 trillion in assets to help reveal the risk in their investment portfolios. In 2003, CDP included only 253 reporting institutions, and in 2014 their increase to 5,600, including companies and cities.

CDP proposes four main programs focusing on the firms: climate change, water, supply chain and forest. In addition, two more programs exist: cities and carbon action initiative.

CDP claim: 'Companies that measure their environmental risk are better able to manage it strategically. And those that are transparent and disclose this information are providing decision makers with access to a critical source of global data that delivers the evidence and insight required to drive action'. CDP recognises companies with high-quality disclosure as top scoring companies in the Climate Disclosure Leadership Index (CDLI). This helps firms to get investors and government on their side, and so get the competitive advantage and commercial benefits over their competitors. CDLI has now become a standard, and many investors require the companies not only be reporting to the $\mathrm{CDP}$, but also to have a bounding index position.

\section{DATA}

Our database is unique, and contains personally collected panel data with contribution from South Pole Group. We have built the database by adding several firms' characteristics to an initial database provided by them. Main quantitative information concerning our databases is presented in Table 1.

In total our database contains 135 companies observed over a period of 9 years, from 2005 to 2013. The time period is a very important parameter in our analyses, and so merits closer attention. Ideally, we would use observations over a longer period of time, but the provided data by South Pole Group contains information from 2005 to 2013 only. The driving factor

Table 1: Global database in numbers.

Number of companies: 135

Number of participating companies: 73

Number of non-participating companies: 62

Period: 2005-2013

Regions: EU (48, 29, 19), US (53, 28, 25), UK (34, 16, 18)

Sectors: Consumer Discretionary $(22,10,12)$, Consumer Staples $(16,12,4)$,

Industrials $(36,18,18)$, IT \& Telecommunications $(14,12,2)$,

Energy $(8,3,5)$, Materials $(11,5,6)$, Financials $(12,3,9)$, Health Care $(10,7,3)$, Utilities $(6,3,3)$

Note: In parenthesis you find number of observations for total, participating companies and non-participating companies respectively. 
behind this is the main study variable, a company's greenhouse gas emissions. This highly sensitive information only began to be reported publicly by companies in the past decade, and only in the last few years have a large number of international companies been publishing this information in their annual or other reports. To our knowledge, there is no existing obtainable databases containing company $\mathrm{CO}_{2}$ emissions for a longer period. The companies themselves do not generally hold historical data on their emissions as it is a fairly new measurement, often neglected in the past. As Max Horster, Managing Partner of South Pole Climate Neutral Investments, confirmed: 'It's not that companies are purposely hiding the correct numbers, they just didn't put much effort into it.'

Our data covers three geographic regions: United Kingdom (UK), United States (US) and European Union (EU). This is defined as the country where the company's headquarters are based. Using a range of countries is very important because the origin of the company mostly define a company's policy, including the sustainability. We have chosen these three geographic regions because of their similarities in the corporate social responsibility policy and economic development. The most common regulations in these regions are the EU Emission Trading System, the UK Carbon Reduction Commitment Energy Efficiency Scheme and the US Environmental Protection Agency's Mandatory GHG Reporting Rule. UK is not included in the European union section, because of its specificity in sustainable development strategy.

Moreover, we classify by the Global Industry Classification Standard companies in nine sectors: consumer discretionary, consumer staples, energy, financials, health care, industrials, information technology and telecommunications, materials, utilities.

Our database contains 22 variables capturing company's characteristics for each of nine periods. The main variables with descriptions are presented in the Table 2. Moreover, our data are complete, which means that no missing value is observed for any of the variables.

Table 2: Company's characteristics (variables).

\begin{tabular}{ll}
\hline Variables & Description \\
\hline NAME & Name (nominal); \\
CDP & Reporting to the cdp (binomial) \\
COUNTRY & Headquarter (nominal); \\
SECTOR & Sector (nominal); \\
GHG & CO $_{2}$ emissions in metric tons (digital); \\
R & Revenue in mio chf (digital); \\
GP & Gross profit in mio chf (digital); \\
COGS & Cost of goods sold in mio chf (digital); \\
FA & Fixed assets in mio chf (digital); \\
EMP & Number of employees (digital); \\
P & Share price (digital); \\
RI & Number of employees (digital); \\
KL & Capital labor ratio (digital); \\
GHG-EMP & CO 2 emissions in metric tons per employee (digital); \\
\hline
\end{tabular}




\section{METHODOLOGY: SYNTHETIC CONTROL METHOD}

Synthetic control method (SCM) provides a systematic way to estimate the counterfactual unit so called synthetic control, that is, a convex combination of control units that most closely resemble the treated unit before the treatment in terms of the potential outcome and other relative predictors. The synthetic control allows us to identify the correct counterfactual business-as-usual emissions per employee, versus the actual $\mathrm{CO}_{2}$ emissions per employees for comparison.

Abadie and Gardeazabal [1] introduce the synthetic control method for comparative case study to analyse economic effect of conflict. In two others articles, Abadie et al. [3, 4], the SCM is used to study the efect of anti-tobacco legislation and to examine the economic consequence of political integration. Almer and Winkler [2] introduce the SCM into the environmental problematic, where they evaluate the effect of the commitment to the specific greenhouse gas targets under the Kyoto protocol on the $\mathrm{CO}_{2}$ emissions. In our study, we use the SCM to assess the pertinence of green policy introduction at the business level.

\subsection{Model}

Let $S$ be balanced panel sample of $J+1$ companies indexed by $j=1, \ldots, J+1$. We define that only the first company $j=1$ participates to the CDP and is uninterruptedly exposed to the program after some initial period. This company is described as treated. The rest of the companies are the $J$ potential controls that constitute so called 'donor pool'. It is important to restrict the donor pool to units with outcomes that are thought to be driven by the same structural process as the treated unit. This is the main driver for selecting companies with respect to country and sector.

The units are observed over $T$ periods, indexed by $t=1, \ldots ., T$. We suppose a positive number of pre-intervention periods $T_{0}$, for $t=1, \ldots, T_{0}$, and a positive number of post-intervention periods $T_{1}$, for $t=T_{0}+1, \ldots, T$, with $T=T_{0}+T_{1}$ and $1<T_{0}<T$. In our case $T=9, t=1$ is year 2005 , and $T_{0}=4$ is year 2008. This means that we observe our data between the period 2005-2013 with pre-intervention period 2005-2008.

The variable $Y_{i t}$ measures the impact of the CDP. It is called 'potential outcome' and in our case it is a variable measuring $\mathrm{CO}_{2}$ emissions per employee. Furthermore, $Y_{i t}^{\mathrm{N}}$ is the potential outcome that would be observed for the firm $i$ at time $t$ in the absence of the CDP, and $Y_{i t}^{\mathrm{I}}$ is the $\mathrm{CO}_{2}$ emissions per employee that would be observed for unit $i$ at time $t$. The difference $a_{i t}=Y_{i t}^{I}-Y_{i t}^{N}$ is the effect of the CDP for company $i$ at time $t$, if company $i$ participate to the CDP in periods $t \in\left\{T_{0}+1, \ldots, T\right\}$. Note that the $Y_{i t}^{\mathrm{N}}$ is unobserved for the treated company in the post-intervention period. The synthetic control method aims to construct a synthetic control group providing an estimate for this missing potential outcome.

Ideally, we would like to construct a synthetic control that most closely resembles the treated unit in all relevant pre-intervention characteristics. Abadie and Gardezabal [1] propose to make use of the observed characteristics of the units from the donor pool. To construct our synthetic control we define $(J \times 1)$ vector of weights $\mathbf{W}=\left(w_{2}, \ldots, w_{J+1}\right)^{\prime}$ such that $0<w_{j}<1$, for $j=2, \ldots, J+1$, and $\sum_{j=2}^{J+1} w_{j}=1$.

Each scalar wj represents the weight of company $\mathrm{j}$ in the synthetic control. Each particular vector $\mathbf{W}$ generates one particular weighted average of control companies, therefore one potential synthetic control. Among the set of all possible $\mathbf{W}$, we choose $\boldsymbol{W}^{*}=\left(w_{2}^{*}, \ldots, w_{J+1}^{*}\right)^{\prime}$ such that the resulting synthetic control best approximates first the company exposed to the 
CDP with respect to $\mathrm{CO}_{2}$ emissions per employee in the pre-intervention period and second all other relevant characteristics, $\mathbf{Z}=(R, G P, C O G S, K L, E M P, G H G)$, described in Table 2. In other words we seek $\mathbf{W}^{*}$ such that:

$$
\sum_{j=2}^{J+1} w_{j}^{*} Y_{j t}=Y_{1 t} \quad \text { and } \quad \sum_{j=2}^{J+1} w_{j}^{*} \boldsymbol{Z}_{j t}=\boldsymbol{Z}_{1 t}, \quad \forall t<T_{0} .
$$

Notice that $\sum_{J=2}^{J+1} w_{j}^{*} Y_{j t}$ for $t>T_{0}$ is an estimate for the unobserved counterfactual businessas-usual emissions per employee $Y_{i t}^{N}$; hence, we can easily estimate the treatment effect as:

$$
\hat{a}_{i t}=Y_{1 t}^{I}-\sum_{j=2}^{J+1} w_{j}^{*} Y_{j t}, \quad \forall t>T_{0} .
$$

Abadie et al. [3] resolves the problem of finding $\mathbf{W}^{*}$ by minimising the distance:

$$
\left\|\mathbf{X}_{1}-\mathbf{X}_{0} \mathbf{W}\right\|_{V}=\sqrt{\left(\mathbf{X}_{1}-\mathbf{X}_{0} \mathbf{W}\right)^{\prime} \mathbf{V}\left(\mathbf{X}_{1}-\mathbf{X}_{0} \mathbf{W}\right)}
$$

subject to $0<w_{j}<1, j=2, \ldots, J+1$ and $\sum_{j=2}^{J+1} w_{j}=1$, where $\mathbf{X}_{1}$ denotes a vector of preintervention characteristics of the treated company, including also pre-intervention emissions per employee; $\mathbf{X}_{0}$ denotes a matrix of the same variables for the $J$ companies in the donor pool; $\mathbf{V}$ is some symmetric and positive semidefinite matrix.

\subsection{Statistical inferences}

The use of statistical inference in comparative case study is difficult because of the smallsample nature of the data, the absence of randomisation or lack of a probabilistic sampling to select sample units. These limitations complicate the application of traditional approaches to statistical inference.

Synthetic control method provides the building blocks for an alternative mode of qualitative and quantitative inferences. The SCM systemises the process of estimating the counterfactuals and enables us to conduct falsification exercises, so called 'placebo studies'. Another way to measure and test the misspecification of the model is to use the root mean squared prediction error (RMSPE).

First, the idea of placebo studies is to predict the counterfactual outcome path for the units in the donor pool. This alternative model of inference is supported by the confidence that we have in the treatment effect produced by the synthetic control estimate. We assume that a particular synthetic control estimate reflects the impact of the treatment, and that this impact would be subverted if we obtained estimated effects of similar or even greater magnitude in cases where the intervention didn't take place. In our study we apply so called in-space multiple placebo tests, where we implement synthetic control methods to all controls in the donor pool. Once we get the placebo effects, we can construct the p-value. The p-value is the fraction of effects greater or equal to the effect estimated for the unit of interest. In this case, the p-value represents the probability of obtaining an estimate at least as large as the one obtained for the unit of interest when the intervention is reassigned at random in the data set.

The second measure to evaluate the estimates, RMSPE, calculates, as stated by Abadie et al. [4], 'the lack of fit between the path of the outcome variable for any particular unit and 
its synthetic counterpart'. The pre- intervention RMSPE for the treated company under investigation is defined as:

$$
\operatorname{RMSPE}=\left(\frac{1}{T_{0}} \sum_{t=1}^{T_{0}}\left(Y_{1 t}-\sum_{j=2}^{J+1} w_{j}^{*} Y_{j t}\right)^{2}\right)^{1 / 2}
$$

Note that the RMSPE can be defined for any units or time periods. Large pre-intervention RMSPE means the synthetic control doesn't match the treated unit in pre-intervention period. Large post-intervention RMSPE is not indicative of large effect of the intervention if the preintervention RMSPE is also large. Large RMSPE-ratio, that is ratio between post-intervention RMSPE and pre-intervention RMSPE, indicates large treatment effect for a given unit. Its p-value gives us proportion of units with higher RMSPE-ratio to total number of tested units. For inference analyses Abadie et al. [4] recommend to use the units from the donor pool with RMSPE that is smaller than three times RMSPE of the unit under investigation. In our study, we used five times RMSPE rule.

\section{RESULTS}

Our data, restricted to the industrial sector, contain 18 treated and 18 control companies. We observed three extremely large treated companies where we didn't find a matching synthetic control. Their pre-intervention RMSPE was higher than 180. We have removed these from our analysis. The rest of the companies performed relatively well and got the pre-intervention RMSPE lower than 3. Out of the remaining 15 companies, eight companies show decrease of $\mathrm{CO}_{2}$ emissions per employee over the post-intervention period. But our statistical inferences put into evidence the positive treatment effect for only three companies.

Table 3 shows inference test results for the eight companies with a positive average treatment effect. ABM Industries shows a very small decrease in $\mathrm{CO}_{2}$ emissions per employee and the placebo p-value confirms the negligible improvement in $\mathrm{CO}_{2}$ emissions. Centrotec Sustai, Flsmith \& CO, Kobenhavns Lufth and Kone Oyjb show relatively good decreases in their $\mathrm{CO}_{2}$ emissions per employee, but relatively small RMSPE-ratios, while their high p-values indicate no improvement from the pre-treatment period. For these companies we couldn't approve with placebo tests and RMSPE-ratios a significant and positive treatment effect.

Table 3: Placebo tests results.

\begin{tabular}{lccccc}
\hline & $\begin{array}{c}\text { Treatment } \\
\text { effect }\end{array}$ & RMSPE & RMSPE-ratio & $\begin{array}{c}\text { RMSPE-ratio } \\
\text { p-value }\end{array}$ & $\begin{array}{c}\text { Placebo } \\
\text { p-value }\end{array}$ \\
\hline Abm industries & -0.24 & 0.05 & 6.67 & 0.11 & 0.50 \\
Atlantia spa & -4.18 & 0.45 & 11.59 & 0.26 & 0.07 \\
Centrotec sustai & -0.76 & 2.97 & 0.25 & 1.00 & 0.31 \\
Flsmith \& co & -2.13 & 1.24 & 1.77 & 0.79 & 0.11 \\
Kobenhavns lufth & -3.59 & 1.62 & 2.22 & 0.47 & 0.05 \\
Kone oyjb & -1.09 & 0.04 & 2.30 & 0.74 & 0.36 \\
Obracson huarte & -3.72 & 0.33 & 14.57 & 0.21 & 0.06 \\
Serco group & -1.57 & 0.08 & 19.19 & 0.10 & 0.18 \\
\hline
\end{tabular}


(a) Path plots
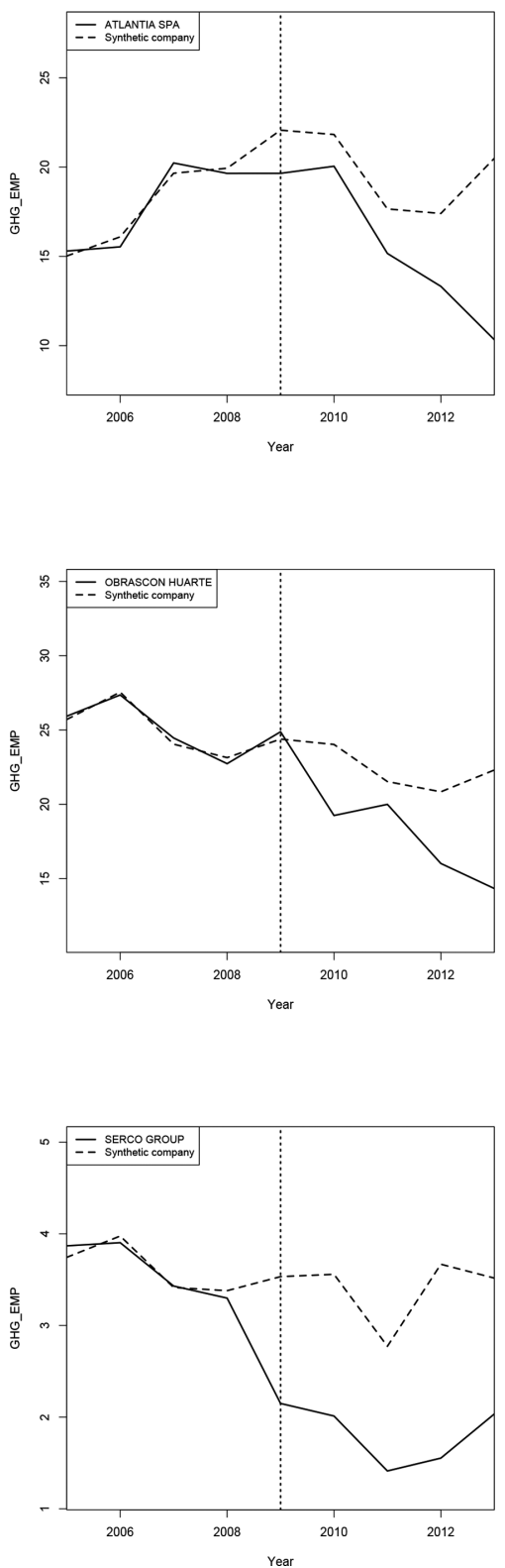

(b) Placebo plots
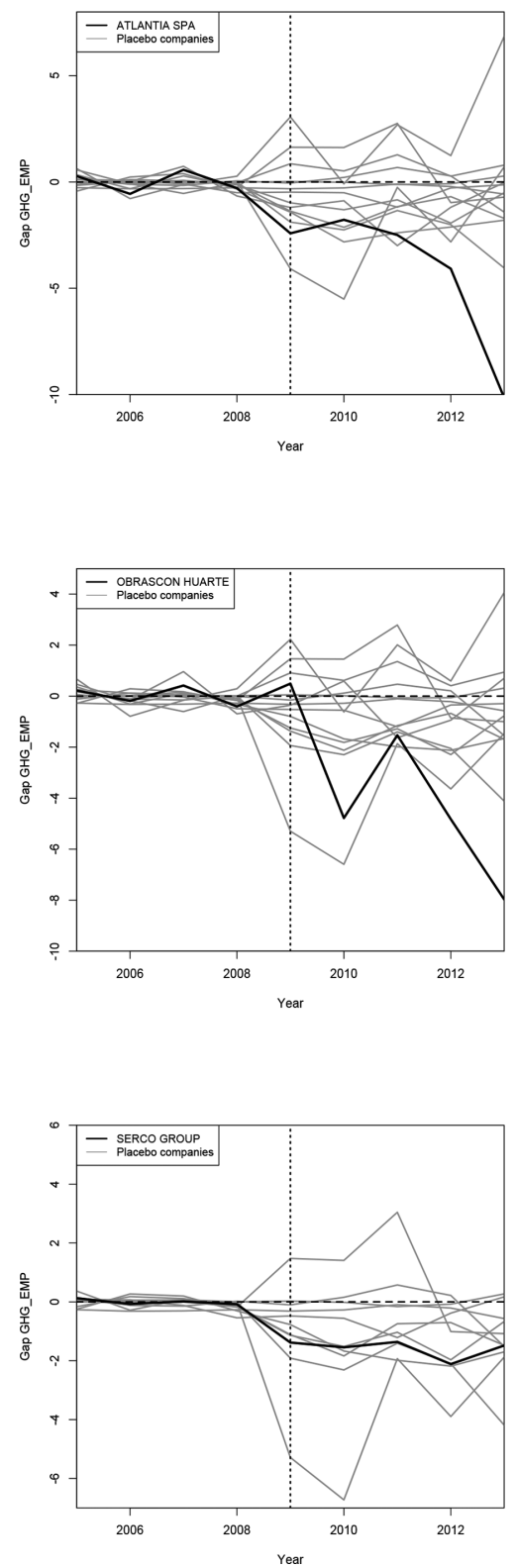

Figure 1: (a) Synthetic matching and (b) permutation tests for Atlantia S.p.A., Obracson Huarte and Serco Group.

On the other side, Atlantia S.p.A., Obracson Huarte and Serco Group outperform the other companies in the values of the tests. Figure 1 presents the synthetic matching and permutation tests for these three companies. 
The first column of the Fig. 1 shows the graphs of the gaps of the $\mathrm{CO}_{2}$ emissions per employee for the treated companies and their synthetic controls. The almost parallel lines in pre-intervention period, before the vertical line, indicate a good match between the treated company and its synthetic control with respect to the $\mathrm{CO}_{2}$ emissions per employee. This result is approved by small pre-intervention RMSPE for all three companies. The gap between the treated and synthetic control in post-intervention period indicates treatment effect, the bigger the gap, the larger the effect. We observe positive treatment effects, supported by high RMSPE-ratios, indicating large decreases in $\mathrm{CO}_{2}$ emissions per employee in the post-intervention period for the three companies.

The second column of the Fig. 1 shows the treated units and their relative placebo treated units. We can observe almost all placebo treated units sitting above the treated units under investigation. This means that the positive treatment effect of the treated unit is not random. The results are approved by both low placebo and RMSPE p-values for all three companies, showing that other placebo treated companies didn't perform as well as the treated companies under investigation.

\section{CONCLUSIONS}

The objective of our study was to assess the pertinence of green policy introduction at the business level. In particular, we intended to evaluate whether signing up to the CDP, has a positive effect on companies' emissions.

In order to perform our analyses we made use of the synthetic control methods that allowed us to not only get the treatment effect for each of the studied companies, but also to perform statistical inferences. We can notice that this method is well suited to our study and can be used for further similar research, as for exemple to analyse whether the CDP firms really achieve their target for $\mathrm{CO}_{2}$ emissions.

For the firms from the industrial sector, we found that out of the 14 treated units only three of them show significant and positive treatment effects. This result could be due to relatively short post-treatment period or to the fact that other non-participating companies are also under good regulation of $\mathrm{CO}_{2}$ emissions.

We can conclude that the effect on emissions of reporting to the CDP varies on a case by case basis, and that with the exception of three companies we found little evidence for a general significant positive treatment effect.

\section{REFERENCES}

[1] Abadie, A. \& Gardeazabal, J., The economic costs of conflict: a case study of the basque country. The American Economic Review, 93, pp. 113-132, 2003. http://dx.doi.org/10.1257/000282803321455188

[2] Almer, C. \& Winkler, R., The effect of kyoto emission targets on domestic co2 emissions: a synthetic control approach. Technical report, Uni-versitaet Bern, Departement Volkswirtschaft, 2012.

[3] Abadie, A., Diamond, A. \& Hainmueller, J., Synthetic control methods for comparative case studies: Estimating the effect of california's tobacco control program. Journal of the American Statistical Association, 150, pp. 493-505, 2010. http://dx.doi.org/10.1198/jasa.2009.ap08746

[4] Abadie, A., Diamond, A. \& Hainmueller, J., Comparative politics and the synthetic control method. American Journal of Political Science, 59, pp. 495-510, 2015. http://dx.doi.org/10.1111/ajps.12116 